\title{
Modulation of colonic barrier function by the composition of the commensal flora in the rat
}

\author{
A García-Lafuente, M Antolín, F Guarner, E Crespo, J-R Malagelada
}

\begin{abstract}
Background and aims-Altered intestinal permeability is a key pathogenetic factor of idiopathic bowel inflammation. We investigated in the rat if changes in the composition of the bowel flora can alter colonic permeability.

Methods-A colonic segment was surgically excluded from faecal transit and brought out as a loop to the abdominal wall through two colostomies. The loop was used for colonisation with specific bacterial strains after eradication of the native flora with antibiotics. Lumen to blood clearance of dextran (molecular weight 70 000) and mannitol (molecular weight 182) was measured in rats recolonised with a single bacterial strain from rat colonic origin, and in control rats whose colonic loop was kept free of bacteria by antibiotics. Actual colonisation was confirmed by culture of segment effluents. Results-Colonisation with Escherichia coli, Klebsiella pneumoniae, and Streptococcus viridans significantly increased lumen to blood clearance of mannitol. Colonisation with Lactobacillus brevis had the opposite effect and reduced permeability to mannitol. Bacteroides fragilis did not induce significant changes. Permeability to dextran was not altered by any of the strains tested.
\end{abstract}

Conclusions-Certain commensal bacteria can modify colonic wall permeability to luminal substances.

(Gut 2001;48:503-507)

Keywords: bacteria; dextran; intestinal permeability; mannitol; rat

Digestive System

Research Unit,

Hospital General

Universitari Vall

d'Hebron, Barcelona

08035, Spain

A García-Lafuente

M Antolín

F Guarner

J-R Malagelada

Microbiology Unit, Hospital General Universitari Vall d'Hebron, Barcelona, Spain

E Crespo

Correspondence to: Dr F Guarner.

fguarnera@meditex.es

Accepted for publication 31 October 2000

The colonic mucosal barrier is a complex physicochemical structure that separates the internal "milieu" from a polluted luminal environment. Barrier function ultimately depends on the integrity of the mucosa-from the endothelium to the epithelial cell lining-and the reactivity of dynamic defensive factors such as mucosal blood flow and epithelial secretions. A gel formed by the interaction of various mucosal secretions, including mucin glycoproteins, trefoil peptides, and surfactant phospholipids, comprises the mucus layer that covers the epithelium. ${ }^{1-3}$ This layer gives hydrophobic properties to the surface and prevents the influx of water soluble bacterial products and toxins. ${ }^{4}$ A large variety of bacterial populations with different pathogenic potentials and a high concentration of chemical and bacterial toxins are constantly challenging the structure and function of the colonic mucosal barrier.

Morphological or functional derangement of the mucosal barrier may lead to changes in intestinal permeability. Clinical studies have shown altered intestinal permeability in a number of disorders, including intestinal infections, Crohn's disease, coeliac sprue, food intolerance, and non-steroidal antiinflammatory drug induced enteropathy. ${ }^{5}$ Increased intestinal permeability has also been observed in relatives of patients with Crohn's disease $^{6}$ and in patients in remission. ${ }^{7}$ These observations led to a proposal that the permeability defect may be a key pathogenetic factor of idiopathic bowel inflammation as it facilitates mucosal penetration of proinflammatory antigens that challenge the immune system. In several conditions, including Crohn's disease, ulcerative colitis, and coeliac disease, mucosal overproduction of immunoglobulin G antibodies reflects a state of hyperresponsiveness to usually harmless luminal antigens. ${ }^{89}$

Previous experimental work from our laboratory showed that colonising bacteria may alter colonic barrier function. ${ }^{10}$ We found that colonising bacteria can increase the lumen to blood passage of trinitrobenzenesulphonic acid, a toxic agent widely used for induction of experimental colitis, ${ }^{11}$ and render the mucosa vulnerable to luminal injury. The aim of the current study was to investigate the role of specific bacterial strains from rat colonic origin on intestinal permeability, using a rat model that allows single bacterial colonisation of a bowel loop excluded from faecal transit. For this purpose, we tested lumen to blood clearance of two probes (dextran, as a large molecular size probe, and mannitol, as a small molecular size probe) in rats colonised with a single bacterial strain.

\section{Methods}

ANIMALS

Specific pathogen free male Sprague-Dawley rats weighing 200-250 g were purchased from Centre d'Elevage $\mathrm{R}$ Janvier (Le Genest, France). The rats were housed under controlled temperature, humidity, and illumination conditions, and maintained on standard rodent chow (Letica, Barcelona, Spain) and tap water. The local research committee (Comissio de Recerca, Hospital General Universitari Vall d'Hebron) approved all studies.

EXPERIMENTAL MODEL

Rats with a colonic loop excluded from faecal transit were used. The excluded colonic loop provides an in vivo ecological environment for colonisation with preselected bacterial strains, 
after elimination of the native flora with antibiotics. The colonic loop was surgically prepared as previously described. ${ }^{12}$ In anaesthetised rats (90 mg/kg ketamine, Parke-Davis, Ann Arbour, Michigan, USA, and $4 \mathrm{mg} / \mathrm{kg}$ xylazine, Bayer, Leverkusen, Germany), a colonic segment (ascending to the mid-descending colon) was brought out to the anterior abdominal wall through two colostomies. The caecum was removed, and continuity of the tract was achieved by anastomosis of the terminal ileum to the distal colon. After suture of the abdominal wall, colostomies were washed with broad spectrum non-absorbable antibiotics $(5 \mathrm{mg} / \mathrm{ml}$ imipenem and $5 \mathrm{mg} / \mathrm{ml}$ vancomycin in sterile saline) and closed by ligature to prevent the entry of air into the colonic segment. Thereafter, rats were allowed to recover, and standard chow pellets and water were provided ad libitum. By diversion of faecal transit, bacterial populations within the loop can be controlled and maintained without significant variations for several days. ${ }^{12}$

EXPERIMENTAL DESIGN

Six groups of rats were surgically prepared as described above. In the control group, the colonic segments were flushed through the colostomies with the solution of broad spectrum antibiotics (imipenem and vancomycin, as above) on days 1 and 4 after surgery, under anaesthesia. The remaining five groups were used for recolonisation with a single bacterial strain. All strains were of rat intestinal origin and included: Escherichia coli, Klebsiella pneumoniae, Streptococcus viridans, Bacteroides fragilis, and Lactobacillus brevis. These bacterial strains were identified by standard bacteriological procedures, including biochemical testing with API strips and Vitek system identification cards (bioMérieux, Marcy-l'Etoile, France), and had been used in previous experimental studies using the same model. ${ }^{1012} 13$ Colonic segments were recolonised 24 hours after surgery. Under anaesthesia, colostomies were flushed with the antibiotic mix and kept tightly closed for two hours. Thereafter, colostomies were washed with sterile saline, inoculated with $1.5-2.0 \mathrm{ml}$ of a specific bacterial suspension, and immediately closed by ligature. Bacterial suspensions were prepared from liquid cultures of each individual strain and used after the phase of logarithmic growth, containing approximately $10^{10}$ colony forming units/ml. Four days after recolonisation, segment washings with sterile saline were cultured to check actual bacterial colonisation. Only rats with positive culture for the specific strain and without other contaminant bacteria were used for the permeability studies. All rats given antibiotics on days 1 and 4 after surgery showed negative cultures of segment washings and were included in the control group.

COLONIC PERMEABILITY

Lumen to blood clearance of ${ }^{14} \mathrm{C}-\mathrm{D}-\mathrm{mannitol}$ (molecular weight $184 \mathrm{Da}$; Amersham International, Buckinghamshire, UK) and ${ }^{3} \mathrm{H}-$ dextran (molecular weight $70000 \mathrm{Da}$; Amersham) was measured in groups of control and recolonised rats (controls $\mathrm{n}=10$; Streptococcus viridans $\mathrm{n}=6$; Klebsiella pneumoniae $\mathrm{n}=6$; Escherichia coli $\mathrm{n}=7$; Bacteroides fragilis $\mathrm{n}=7$; Lactobacillus brevis $\mathrm{n}=9$ ). Four days after bacterial inoculation, rats were anaesthetised (90 $\mathrm{mg} / \mathrm{kg}$ ketamine and $4 \mathrm{mg} / \mathrm{kg}$ xylazine), a tracheotomy performed, and the right carotid artery and left jugular vein were cannulated with polyethylene tubing (PE-50; Clay Adams, Becton Dickinson, Parsippany, New Jersey, USA). Bilateral nephrectomy was achieved by ligation of the renal pedicles through right and left lumbotomy. A $500 \mu \mathrm{l}$ bolus of $10 \mathrm{mmol} / \mathrm{ml}$ cold D-mannitol in saline with $0.4 \mu \mathrm{Ci}$ $\mathrm{D}-\left[1-{ }^{14} \mathrm{C}\right]$ mannitol and $0.3 \mu \mathrm{Ci}\left[{ }^{3} \mathrm{H}\right]$ dextran was instilled into the lumen of the excluded colonic segment and both colostomies were kept closed for five hours. Total radioactivity administered to each animal was calculated by counting two $50 \mu \mathrm{l}$ aliquots of the administered solution using a scintillation counter with a dual channel programme for simultaneous ${ }^{14} \mathrm{C}$ and ${ }^{3} \mathrm{H}$ counting (LS 6500; Beckman Instruments, Palo Alto, California, USA). Blood samples $(500 \mu \mathrm{l})$ were taken through the carotid line at $0,1,2,3,4$, and 5 hours after the intracolonic bolus, and used for measurement of plasma radioactivity levels by means of a scintillation counter. Immediately after sampling, an equal volume of $2 \%$ albumin in sterile saline was administered via the jugular catheter to prevent changes in haemodynamics. Finally, the rat was killed by anaesthetic overdose, and two urine samples were obtained from the bladder and counted to exclude leakage of the radioactive probes. Accumulation of the probes in blood was assumed to reflect entry rates (lumen to blood clearance) of the molecules as rats had been nephrectomised and the compounds are not metabolised. Thus data on lumen to blood clearance of D-mannitol were expressed as a percentage of the administered dose that accumulated in the extracellular fluid volume $(25 \%$ of body weight), normalised by the length $(\mathrm{cm})$ of the excluded colonic segment of each rat. Lumen to blood clearance of dextran was expressed as a percentage of the administered dose that accumulated in the intravascular fluid volume ( $6 \%$ of body weight), normalised by the length of the excluded colonic segment.

Ancillary experiments with controls $(n=8)$ and $E$ coli recolonised rats $(\mathrm{n}=5)$ used ${ }^{51} \mathrm{Cr}$ EDTA (molecular weight 341 Da; Dupont, NEN Research Products, Boston, Massachusetts, USA) as a permeability probe as this compound is biologically inert and cannot be degraded by bacteria. The experiments were performed following the same procedure as with mannitol and dextran. A $500 \mu \mathrm{l}$ bolus of $0.5 \mathrm{mmol} / \mathrm{ml}$ cold EDTA in saline with $5 \mu \mathrm{Ci}$ ${ }^{51} \mathrm{Cr}-\mathrm{EDTA}$ was instilled into the lumen of the excluded colonic segment and both colostomies were kept closed for five hours. Radioactivity in two aliquots of the administered dose and in hourly blood samples was measured by means of a gamma counter. Lumen to blood clearance of ${ }^{51} \mathrm{Cr}$-EDTA was expressed as a percentage of the administered dose that accumulated in the extracellular fluid 
volume ( $25 \%$ of body weight), normalised by the length of the excluded colonic segment.

\section{MICROBIOLOGICAL METHODS}

Bacterial suspensions for inoculations were prepared from cultures of each individual species. All strains were isolated from inmate Sprague-Dawley rats and maintained at $-80^{\circ} \mathrm{C}$. Bacteria were defrosted and allowed to grow in liquid medium (brain-heart infusion with haemin and vitamin $\mathrm{K}_{1}$ ) at $37^{\circ} \mathrm{C}$ until the logarithmic growth phase. The final inoculation suspension contained approximately $10^{10}$ colony forming units/ml.

Saline washings of the excluded colonic segment were incubated under aerobic and anaerobic conditions to check actual bacterial colonisation. Aerobic media consisted of blood agar, blood agar with nalidixic acid, McConkey agar, Cled agar, and Sabouraud agar. Anaerobic media consisted of lacquered blood agar enriched with haemin and vitamin $\mathrm{K}_{1}$, blood agar with phenyl-ethanol, and agar-aztreonam $(20 \mu \mathrm{g} / \mathrm{ml})$. Plates inoculated for obligate anaerobes were incubated in an anaerobic chamber for $48-72$ hours at $37^{\circ} \mathrm{C}$, and plates for aerobes in air at $37^{\circ} \mathrm{C}$.

\section{STATISTICAL METHODS}

Results are expressed as mean (SEM). Statistical differences between groups were determined using overall analysis of variance, and the Student-Newman-Keuls method as posttest.

\section{Results}

Colonic permeability to the large molecular size probe dextran was very low in all groups of rats. Figure 1 shows lumen to blood clearance of dextran five hours after intracolonic administration of the probe. No differences were found between rats recolonised with a single bacterial strain and the control rats that were given antibiotics to prevent bacterial colonisation.

In contrast, permeability to the small molecular size probe mannitol varied considerably among the six groups of rats. As shown in fig 2,

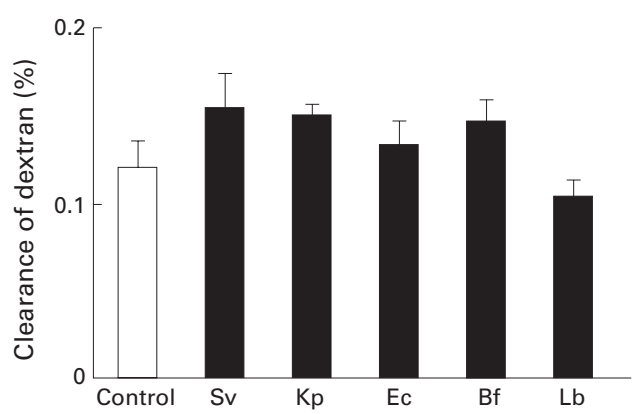

Figure 1 Lumen to blood clearance of dextran five hours after administration of the labelled probe into the excluded colonic segment of controls $(n=10)$ and rats recolonised with a single bacterial strain (Sv, Streptococcus viridans, $n=6 ;$ Kp, Klebsiella pneumoniae, $n=6 ;$ Ec, Escherichia coli, $n=7 ; B f$, Bacteroides fragilis, $n=7$; and L $b$, Lactobacillus brevis, $n=9)$. Clearance is expressed as a percentage of the administered dose that accumulated in the intravascular space and normalised by length of the excluded colonic segment. Values are mean (SEM). No significant differences were found.

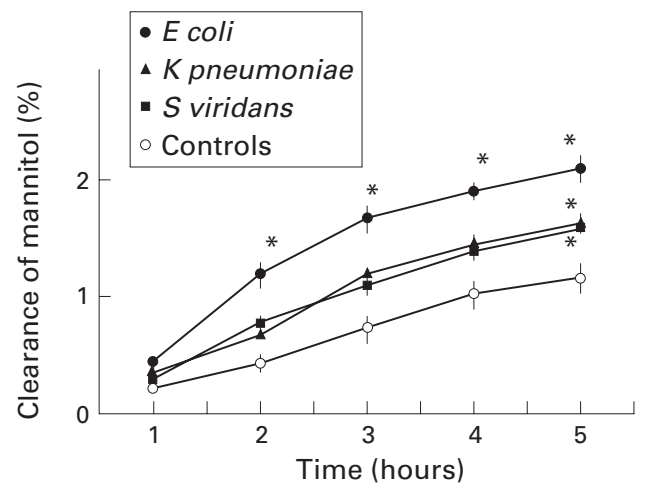

Figure 2 Lumen to blood clearance of mannitol in controls $(n=10)$ and in rats recolonised with a single bacterial strain (Streptococcus viridans, $n=6$; Klebsiella pneumoniae, $n=6$; Escherichia coli, $n=7)$. Clearance is expressed as a

percentage of the administered dose that accumulated in the extracellular space and normalised by length of the colonic segment. Values are mean (SEM). ${ }^{\star} p<0.05$ compared with controls at same time point.

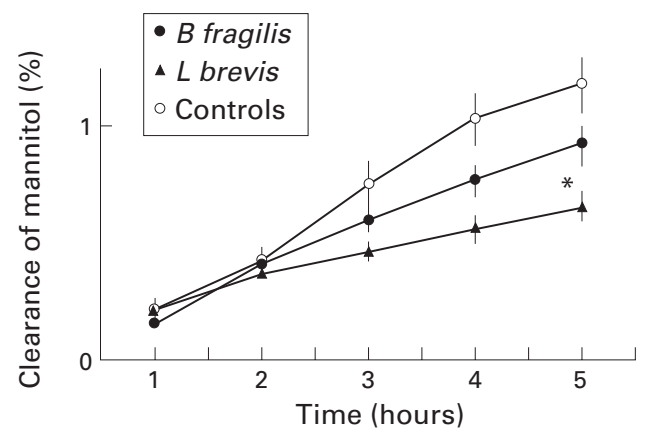

Figure 3 Lumen to blood clearance of mannitol in controls $(n=10)$ and in rats recolonised with a single bacterial strain (Bacteroides fragilis, $n=7$; Lactobacillus brevis, $n=9$ ).

Values are mean (SEM). ${ }^{*} p<0.05$ compared with controls at same time point.

colonisation with the strains $E$ coli, Klebsiella pneumoniae, and Streptococcus viridans increased colonic wall permeability to mannitol compared with control non-colonised rats. The effect of $E$ coli was more intense than that of the other two strains, and the increased entry rate of mannitol was significant at early time points of the experiment. Colonisation with Bacteroides fragilis or Lactobacillus brevis reduced the passage of mannitol from the colonic lumen to the blood but only the effect of the lactobacillus strain was statistically significant (fig 3 ).

The effect of colonisation with $E$ coli on lumen to blood clearance of EDTA was tested to confirm the bacterial effect on permeability

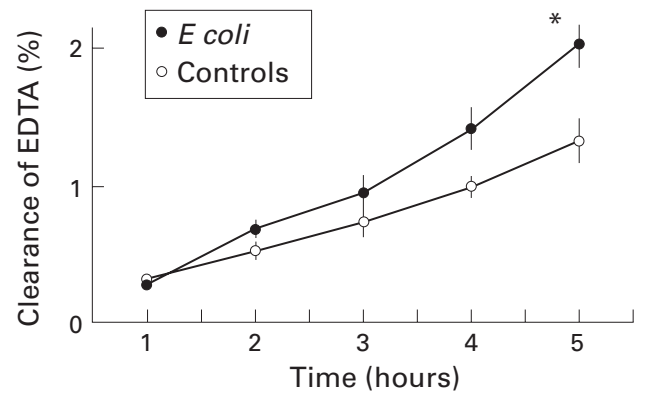

Figure 4 Lumen to blood clearance of ${ }^{51} \mathrm{Cr}$-EDTA in controls $(n=8)$ and in rats recolonised with Escherichia coli $(n=5) .{ }^{*} p<0.05$ compared with controls at same time point. 
using an alternative probe. As shown in fig 4, recolonised rats showed significantly higher accumulation of the probe than control rats.

\section{Discussion}

Our study investigated the effect of colonising bacteria on colonic barrier function. We tested the effect of five bacterial strains of rat intestinal origin in a model allowing in vivo single strain colonisation of a bowel loop excluded from faecal transit. None of the bacteria used in this study induced changes in colonic permeability to the large molecular size probe dextran. The large size of this probe precludes its passage through either transcellular or paracellular pathways under normal conditions. Hence our data suggest that colonisation with these particular strains did not induce epithelial dysfunction or damage compared with non-colonisation. However, the experiments using the small molecular size probe mannitol showed significant differences between strains. Some strains increased colonic permeability to mannitol and among them, $E$ coli was particularly active. In contrast, Lactobacillus brevis reduced intestinal permeability to mannitol. We conclude from our data that some commensal bacteria may impair colonic barrier function and increase colonic permeability to luminal toxins, whereas other species may improve the tightness of the barrier.

Mannitol is a sugar alcohol of small molecular weight $(184 \mathrm{Da})$ and size $(6.5 \AA)$ that is not metabolised by mammalian tissues. However, bacterial metabolism of mannitol cannot be excluded. Bacterial degradation of this sugar could yield small metabolites bearing the ${ }^{14} \mathrm{C}$ label and enhance the passage of the label through the colonic wall without actual changes in permeability. We did not perform specific experiments to assay mannitol metabolism by the bacterial strains used in our study. According to the data obtained in vitro with bacteria identification cards, only the $E$ coli strain can metabolise mannitol. However, metabolic activities may differ considerably between the in vitro and in vivo situation. Mannitol has been used to test the effect of $E$ coli on epithelial permeability by other investigators. ${ }^{14}$ We conducted ancillary experiments using ${ }^{51} \mathrm{Cr}$-EDTA as a permeability probe that cannot be degraded by bacteria. The molecular weight (341 Da) and size (10.5 $\mathrm{A}$ ) of EDTA are larger than that of mannitol but the ${ }^{51} \mathrm{Cr}$-EDTA probe has been shown to permeate the intestinal epithelial barrier through paracellular junctions under normal conditions. ${ }^{5}$ Our experiments confirmed that $E$ coli colonisation may alter colonic barrier function as lumen to blood clearance of ${ }^{51} \mathrm{Cr}$-EDTA was increased.

The effect of adherent bacteria on transepithelial electrical resistance has been investigated by Spitz and colleagues ${ }^{15}$ who observed in the rat that dexamethasone treatment increased counts of bacteria adherent to the intestinal mucosa and decreased transepithelial electrical resistance. These changes were more marked in the ileum, caecum, and colon, and antibiotic treatment prevented the effect of dexamethasone on electrical resistance. These findings are consistent with the concept that adherent bacteria may increase intestinal permeability. Further experiments by the same authors ${ }^{14}$ demonstrated in vitro that adherence of enteropathogenic $E$ coli to intestinal epithelial cell monolayers disrupts the paracellular tight junctions. Moreover, other investigators have shown that enterotoxin from Bacteroides fragilis decreases transepithelial electrical resistance and opens up the spaces between enterocytes in confluent cell cultures of human intestinal epithelial cells. ${ }^{16}$ Further studies with strains of Bacteroides fragilis that produce enterotoxin (fragilysin) have demonstrated that the enterotoxin increases epithelial permeability to mannitol in human colonic mucosa mounted in Ussing chambers. ${ }^{17}$ This study also observed that the enterotoxin damages crypts and surface colonocytes of the human colonic epithelium. ${ }^{17}$ In contrast with these previous investigations, we observed that $E$ coli can increase colonic permeability without producing any damage at the epithelial level, as assessed by the dextran permeability test. Our study suggests that commensal non-pathogenic bacteria may induce significant changes in colonic permeability.

Other experimental studies have shown that certain strains of lactobacillus may prevent or reverse intestinal permeability disorders. For instance, prolonged cow's milk challenge in suckling rats increases gut permeability to intact proteins, and oral bacteriotherapy with the Lactobacillus casei strain GG counteracts the increase in intestinal permeability. ${ }^{18}$ Furthermore, oral administration of Lactobacillus plantarum or Lactobacillus reuteri reduced the severity of intestinal permeability changes induced by methotrexate in rats. ${ }^{19}$ In our study, we found that colonisation with a Lactobacillus brevis strain slightly reduced colonic permeability to mannitol in rats with a normal colonic barrier function. Our findings are, therefore, consistent with these previous observations and further support the concept that the intestinal barrier may be improved by certain lactobacilli strains in the bowel lumen. It is becoming clear that commensal bacteria can influence the colonic barrier function in normal non-pathological conditions. Our findings may contribute to the understanding of pathophysiological mechanisms in clinical disorders associated with altered intestinal permeability.

This research was supported by grant SAF 95-187 from Comisión Interministerial para la Investigación Científica y Técnica (Madrid, Spain)

1 Kindon H, Pothoulakis C, Thim L, et al. Trefoil peptide protection of intestinal epithelial barrier function: cooperative interaction with mucin glycoprotein. Gastroenterology 1995;109:516-23

2 Matsuo K, Ota H, Akamatsu T, et al. Histochemistry of the surface mucous gel layer of the human colon. Gut 1997;40: $782-9$.

3 Lichtenberger LM. The hydrophobic barrier properties of gastrointestinal mucus. Annu Rev Physiol 1995;57:565-83.

4 Lugea A, Salas A, Casalot J, et al. Surface hydrophobicity of the rat colonic mucosa is a defensive barrier against macromolecules and toxins. Gut 2000;46:515-21.

5 Bjarnason I, Macpherson A, Hollander D. Intestinal permeability: an overview. Gastroenterology 1995;108: 1566-81.

6 Hilsden RJ, Meddings JB, Sutherland LR. Intestinal permeability changes in response to acetylsalicylic acid in relatives of patients with Crohn's disease. Gastroenterology 1996;110: of patients 
7 Wyatt J, Vogelsang H, Hübl W, et al. Intestinal permeability and the prediction of relapse in Crohn's disease. Lancet 1437-9.

8 Brandtzaeg P, Halstensen TS, Kett K, et al. Immunobiology and immunopathology of human gut mucosa: humoral immunity and intraepithelial lymphocytes. Gastroenterology 1989;97:1562-84.

9 Macpherson A, Khoo UY, Forgacs I, et al. Mucosal antibodies in inflammatory bowel disease are directed against intestinal bacteria. Gut 1996;38:365-75.

10 García-Lafuente A, Antolín M, Guarner F, et al. Derangement of mucosal barrier function by bacteria colonizing the rat colonic mucosa. Eur f Clin Invest 1998;28:1019-26.

11 Morris GP, Beck PL, Herridge MS, et al. Hapten-induced model of chronic inflammation and ulceration in the rat colon. Gastroenterology 1989;96:795-803.

12 García-Lafuente A, Antolín M, Guarner F, et al. Incrimination of anaerobic bacteria in the induction of experimental tion of anaerobic bacteria in the induction

13 Mourelle M, Salas A, Guarner F, et al. Stimulation of transforming growth factor- $\beta 1$ by enteric bacteria in the pathogenesis of rat intestinal fibrosis. Gastroenterology 1998;114: genesis of
14 Spitz J, Yuhan R, Koutsouris A, et al. Enteropathogenic Escherichia coli adherence to intestinal epithelial monolayers diminishes barrier function. Am f Physiol 1995;268: G374-9.

15 Spitz J, Hecht G, Taveras M, et al. The effect of dexa-methasone administration on rat intestinal permeability: the role of bacterial adherence. Gastroenterology 1994;106:35-41.

16 Wells CL, Van de Westerlo EMA, Jechorek RP, et al. Bacteroides fragilis enterotoxin modulates epithelial permeability and bacterial internalization by HT-29 enterocytes. Gastroenterology 1996;110:1429-37.

17 Riegler M, Lotz M, Sears C, et al. Bacteroides fragilis toxin 2 damages human colonic mucosa in vitro. Gut 1999;44: 504-10.

18 Isolauri $\mathrm{E}$, Majamaa $\mathrm{H}$, Arvola $\mathrm{T}$, et al. Lactobacillus casei strain GG reverses increased intestinal permeability induced by cow's milk in suckling rats. Gastroenterology 1993;105:1643-50.

19 Mao Y, Nobaek S, Kasravi B, et al. The effects of Lactobacillus strains and oat fiber on methotrexate-induced enterocolitis in rats. Gastroenterology 1996;111:334-44.

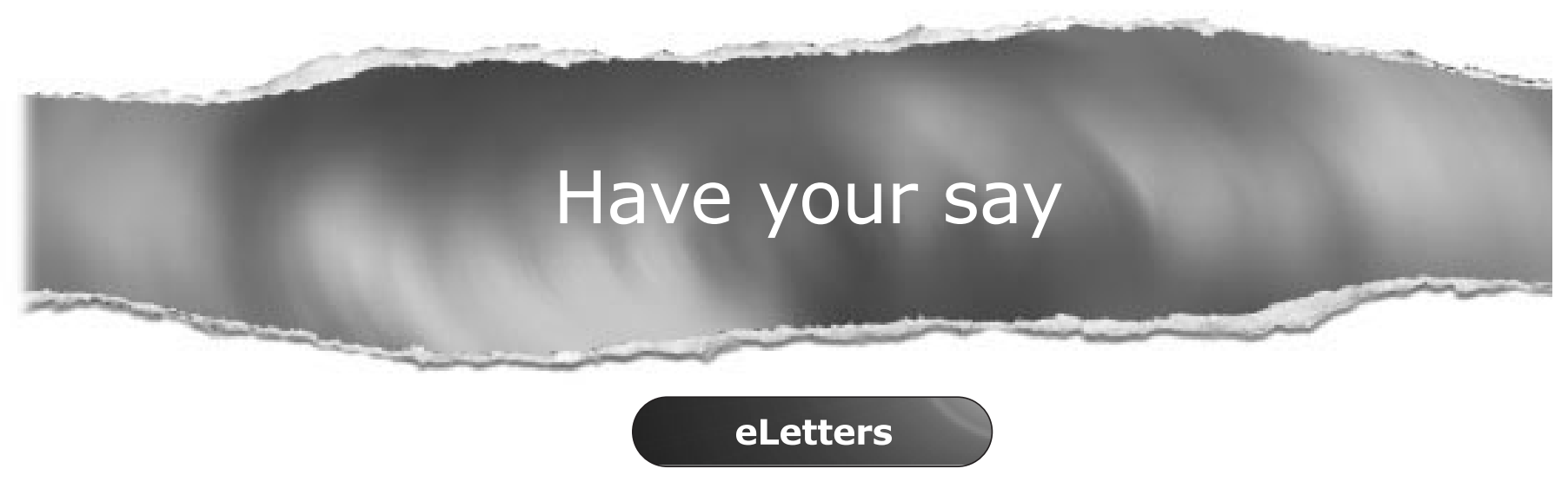

If you wish to comment on any article published in Gut you can send an eLetter using the eLetters link at the beginning of each article. Your response will be posted on

Gut online within a few days of receipt (subject to editorial screening).

\section{www.gutjnl.com}

